\title{
Peripheral blood gene expression patterns discriminate among chronic inflammatory diseases and healthy controls and identify novel targets
}

\author{
Bertalan Mesko+1, Szilard Poliska1+1,4, Andrea Szegedi33, Zoltan Szekanecz5 ${ }^{5}$ Karoly Palatka6, Maria Papp ${ }^{6}$ and \\ Laszlo Nagy*1,2,4
}

\begin{abstract}
Background: Chronic inflammatory diseases including inflammatory bowel disease (IBD; Crohn's disease and ulcerative colitis), psoriasis and rheumatoid arthritis (RA) afflict millions of people worldwide, but their pathogenesis is still not well understood.

It is also not well known if distinct changes in gene expression characterize these diseases and if these patterns can discriminate between diseased and control patients and/or stratify the disease. The main focus of our work was the identification of novel markers that overlap among the 3 diseases or discriminate them from each other.

Methods: Diseased ( $n=13, n=15$ and $n=12$ in IBD, psoriasis and RA respectively) and healthy patients $(n=18)$ were recruited based on strict inclusion and exclusion criteria; peripheral blood samples were collected by clinicians (30 ml) in Venous Blood Vacuum Collection Tubes containing EDTA and peripheral blood mononuclear cells were separated by Ficoll gradient centrifugation. RNA was extracted using Trizol reagent. Gene expression data was obtained using TaqMan Low Density Array (TLDA) containing 96 genes that were selected by an algorithm and the statistical analyses were performed in Prism by using non-parametric Mann-Whitney $U$ test (P-values $<0.05$ ).

Results: Here we show that using a panel of 96 disease associated genes and measuring mRNA expression levels in peripheral blood derived mononuclear cells; we could identify disease-specific gene panels that separate each disease from healthy controls. In addition, a panel of five genes such as ADM, AQP9, CXCL2, IL10 and NAMPT discriminates between all samples from patients with chronic inflammation and healthy controls. We also found genes that stratify the diseases and separate different subtypes or different states of prognosis in each condition.

Conclusions: These findings and the identification of five universal markers of chronic inflammation suggest that these diseases have a common background in pathomechanism, but still can be separated by peripheral blood gene expression. Importantly, the identified genes can be associated with overlapping biological processes including changed inflammatory response. Gene panels based on such markers can play a major role in the development of personalized medicine, in monitoring disease progression and can lead to the identification of new potential drug targets in chronic inflammation.
\end{abstract}

\section{Background}

Chronic inflammatory diseases such as inflammatory bowel disease (IBD; including Crohn's disease - CD and ulcerative colitis - UC), psoriasis and rheumatoid arthritis

\footnotetext{
*Correspondence: nagyl@med.unideb.hu

1 Department of Biochemistry and Molecular Biology, University of Debrecen, Debrecen, Hungary

+ Contributed equally

Full list of author information is available at the end of the article
}

(RA) exist as a substantial burden in social and economic terms worldwide. Despite the importance of these diseases, it is still not clear if characteristic gene expression signatures can discriminate this group of diseases from healthy controls, the various diseases from each other or whether it is possible to stratify the diseases based on gene expression changes.

These chronic conditions have common features such as the autoimmune origin, the frequent co-morbidity and 
a few genes such as IL10, IL23R, SLC22A4 and SLC22A5 that have been identified as contributors to their genetic background [Table 1]. However their prevalence and the tissues affected are clearly different.

RA is a systemic autoimmune disorder, with a prevalence between $0.5-1.0 \%$ [1], that causes inflammation and tissue damage in joints and tendon sheaths. Psoriasis which is a chronic disorder of the skin and joints where the psoriatic plaques are areas of inflammation and excessive skin production, affects approximately $2 \%$ of the population only in the USA [2]. 1-2\% of Western populations suffer from IBD [3] in which the common features are the inflammation of the intestines and the autoimmune origin.

Global and selective gene expression analyses have already been performed in order to gather hints on the mechanisms of these medical conditions by using human biopsy samples such as colon tissue in IBD [4]; skin tissue in psoriasis [5] and synovial tissue biopsy in RA [6]. However peripheral blood is a more accessible source of cells and may be easier to use for screening processes. Furthermore as circulating peripheral blood mononuclear cells
(PBMCs) are key cells of inflammation, it may also reflect disease mechanisms.

In addition studies of gene expression profiling of PMBCs may provide a more cost effective and less invasive alternative to biopsy or invasive measurements [7]. Examples of the clinical implications of this approach include the analysis of human breast cancer progression [8] and PBMC profiles in RA, systemic lupus erythematosus, type I diabetes and multiple sclerosis [9].

It appears therefore that gene expression profiling from PBMCs is a validated tool for discovery and also may be used to explore the pathogenetic background of these medical conditions [10-12]. However comparative studies on the existence of distinct and overlapping gene expression patterns are lacking. We sought to fill this gap by carrying out a comparative analysis of peripheral gene expression patterns of a panel containing 96 genes in various chronic inflammatory diseases and healthy controls.

\section{Methods}

Patient recruitment

The Research Ethics Committee of University of Debrecen Medical and Health Science Center approved the

Table 1: Known SNP - disease associations

\begin{tabular}{|c|c|c|c|}
\hline Gene & IBD & Psoriasis & Rheumatoid arthritis \\
\hline ADAM33 & NA & rs512625 PMID: 18560587 & NA \\
\hline IL10 & rs3024505 PMID: 18836448 & NA & rs1800896 PMID: 18615156 \\
\hline IL13 & NA & rs1800925 PMID: 19554022 & NA \\
\hline IL23R & rs2201841 PMID: 18338763 & rs11209026 PMID: 18369459 & NA \\
\hline IL4 & rs2243250 PMID: 18064451 & NA & NA \\
\hline IL8 & NA & NA & PMID: 18799095 \\
\hline PADI4 & NA & NA & rs2240340 PMID: 12833157 \\
\hline PTGS2 & rs20432 PMID: 16273614 & NA & rs5275 PMID: 18381795 \\
\hline PTPN22 & NA & rs1217414 PMID: 18341666 & rs2476601 PMID: 18466513 \\
\hline SLC22A4 & rs3792876 PMID: 17476680 & rs11568506 PMID: 18614543 & rs3792876 PMID: 15107849 \\
\hline SLC22A5 & rs3792876 PMID: 17476680 & rs2631367 PMID: 16255050 & rs2631367 PMID: 15107849 \\
\hline
\end{tabular}


clinical protocol and the study that were in compliance with the Helsinki Declaration. Signed informed consent was obtained from all healthy and diseased individuals who provided blood sample. Inclusion and exclusion criteria were developed using the best evidence currently available. Online supplement is provided for additional information about inclusion and exclusion criteria [Additional File 1: Figure S2].

The study included 13 patients with IBD; 15 with psoriasis and 12 with RA, all of whom had active disease and were medication-free at the time of blood draw [Table 2]. Blood was also obtained from a group of healthy control individuals (18 patients) that did not show significant differences compared to the diseased groups regarding age. After the subjects fasted overnight for 12 hours, all of the blood samples were obtained locally between 8:00 AM and 9:00 AM; and were processed within one hour after sample collection.

\section{Peripheral blood mononuclear cell collection and RNA processing}

Venous peripheral blood samples were collected by clinicians $(30 \mathrm{ml})$ in Venous Blood Vacuum Collection Tubes containing EDTA (BD Vacutainer K2E). PBMCs were separated by Ficoll gradient centrifugation.

Total RNA was extracted from PBMCs using Trizol reagent (Invitrogen), according to the manufacturer's protocol. RNA quality and quantity were checked on
NanoDrop and Agilent Bioanalyser 2100 (Agilent Technologies).

\section{TaqMan mRNA analysis by RT-QPCR}

Gene expression data was obtained using TaqMan Low Density Array (TLDA) (Applied Biosystems) which is a 384-well micro fluidic card that enables to perform 384 simultaneous real-time PCR runs and which has been used for gene expression profiling in several studies $[13,14]$. This low- to medium-throughput micro fluidic card allows for 2 samples to be run in parallel against 96 TaqMan Gene Expression Assay targets that are preloaded into each of the wells on the card. cDNA was generated with High Capacity cDNA Reverse Transcription Kit according to manufacturer's protocol. 2 micrograms of RNA were used per sample in the RT-PCR runs. $400 \mathrm{ng}$ $(4 \mu \mathrm{l})$ cDNA was used in each sample. $196 \mu$ l nucleasefree water and $200 \mu \mathrm{l} 2 \times$ TaqMan Universal PCR Master Mix (Applied Biosystems) were added for the Real-Time Quantitative PCR measurements. This mixture was then equally divided over four sample-loading ports of the TLDA, each connected to one set of the 96 genes of interest. The arrays were centrifuged once (1', 1300 RPM on room temperature) to equally distribute the sample over the wells. Subsequently, the card was sealed to prevent an exchange between wells. RT-QPCR amplification was performed using an Applied Biosystems Prism 7900HT sequence detection system with the following thermal

Table 2: Patient parameters

\begin{tabular}{|c|c|c|c|c|c|}
\hline Disease Status & Control & \multicolumn{2}{|c|}{ IBD } & Psoriasis & Rheumatoid \\
\hline $\mathrm{n}$ & 18 & \multicolumn{2}{|c|}{13} & 15 & 12 \\
\hline Sex (male/female) & $8 / 10$ & \multicolumn{2}{|c|}{$4 / 9$} & $5 / 10$ & $2 / 10$ \\
\hline Age (years) & $40.07 \pm 21.3$ & \multicolumn{2}{|c|}{$27.92 \pm 8.49$} & $30.53 \pm 9.3$ & $45.83 \pm 18.24$ \\
\hline \multirow[t]{2}{*}{ Clinical subtype } & NA & \multicolumn{2}{|c|}{ CD/UC 8/4 } & Arthritis positive/ & Bone erosion \\
\hline & & CDAI & UCDAI & PASI & DAS28 \\
\hline \multirow[t]{2}{*}{ Clinical severity } & NA & $270.4 \pm 67.18$ & $8 \pm 1.2$ & $27.47 \pm 9.62$ & $6.12 \pm 1.05$ \\
\hline & & $124-365$ & $6-9$ & $15-48$ & $4.07-7.56$ \\
\hline
\end{tabular}

Definition of abbreviations: IBD = Inflammatory bowel disease, $C D=$ Crohn's Disease, CDAI = Crohn's Disease Activity Index, UC = Ulcerative colitis, UC DAI = Ulcerative Colitis Disease Activity Index, PASI = Psoriasis Area Severity Index, DAS28 = Disease Activity Score 28, NA = non available. Data are presented as mean \pm SD; also range in Clinical Severity. 
cycler conditions: $2 \mathrm{~min}$ at $50^{\circ} \mathrm{C}$ and $10 \mathrm{~min}$ at $94.5^{\circ} \mathrm{C}$, followed by 40 cycles of $30 \mathrm{~s}$ at $97^{\circ} \mathrm{C}$ and $1 \mathrm{~min}$ at $59.7^{\circ} \mathrm{C}$.

\section{Gene List Selection Process}

A database containing 400 genes which are associated with the 3 chronic inflammatory diseases and inflammation was constructed by using the data derived from the Human Genome Navigator that lists all the genes related to a specific disease and the evidence the relation is based on; the current medical literature and international databases (e.g. OMIM, Entrez Gene). Genes were also selected from genome-wide association studies as well as microarray analyses focusing on skin biopsy in psoriasis, colon biopsy in IBD and synovial fluid in RA. (Figure 1)

In the second step, genes were put in order by using a score list that was based on the number of publications mentioning the disease-gene association, relation to other diseases and expected expression in PBMC according to previous studies and our pilot experiment in which we analyzed selected genes in PBMC samples through individual RT-QPCR assays. This selection process resulted in 150 genes.

In the third step, genes that did not show expression (missing signal in QPCR measurements) in the pilot study were removed, leading to a final list of 96 genes Additional File 1: Figure S1.

\section{Statistical Analyses}

Relative gene expression levels of each gene were calculated by comparative $\mathrm{Ct}$ method and results were normal-

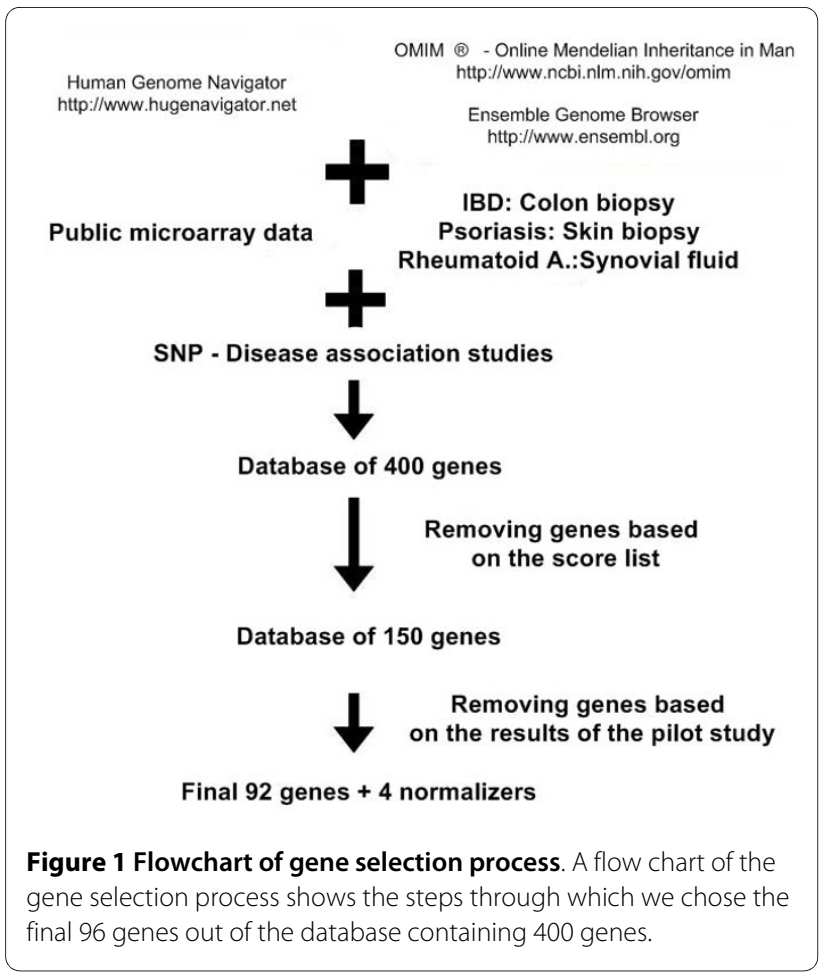

ized to glyceraldehyde-3-phosphate dehydrogenase (GAPDH) expression for each sample. Statistical analyses of the normalized gene expression data were performed in Prism (GraphPad). Due to the fact that our data did not follow normal distributions, the gene expression in groups with different numbers of samples was compared separately using the non-parametric Mann-Whitney U test. P-values $<0.05$ were considered to be statistically significant. This method that does not include correction for multiple comparisons is widely used in the analysis of TLDA data $[15,13]$ and is explained in details in [16].

Gene interactions were analyzed by using the Direct Interactions and Biological Processes functions of GeneSpring GX (Agilent Technologies).

Principal component analysis (PCA), a standard, nonparametric tool that reduces a complex data set to a lower dimension, was used in order to reveal the internal structure of the data sets and to project the differences between diseased and healthy groups based on each set of significantly changing genes.

\section{Results}

Identification of gene panels discriminating chronic inflammatory disease patients from healthy controls

A panel of 96 genes has been selected by the algorithm depicted on Figure 1. Patients were recruited and PBMC gene expression was determined as described in Materials and Methods. When we compared the gene expression results we have found 53 genes that show significant differences between diseased and healthy samples. First, we looked at the gene panels that differentiate each disease from the set of control samples. Altogether 25 genes show significant differences between IBD; 16 genes between psoriasis; 33 genes between RA and controls (Figure 2a-c). Principal component analysis of these gene sets also separates the different groups of samples. In order to get hints of the complex transcriptional basis of inflammatory diseases and to find potential targets that might play a role in the pathogenesis of the conditions, the interaction among multiple genes needs to be revealed. Gene interaction analyses highlighted IFNG, IL4, IL10, MMP9 and TIMP1 in IBD; IL10, IL13 and PTGS2 in psoriasis; IL8, IL10 and PTGS2 in RA. These genes had the most direct interactions that might reveal their key role in the pathogenetic background of the diseases. Gene interaction analysis on each set of genes is provided in the supplement material Additional File 1: Figure S3-5.

\section{Gene sets showing overlapping or differential expression} In order to analyze the gene expression patterns of these conditions, first we created a Venn diagram highlighting those genes that differentiate between a disease and the set of control samples (Figure 3a). 


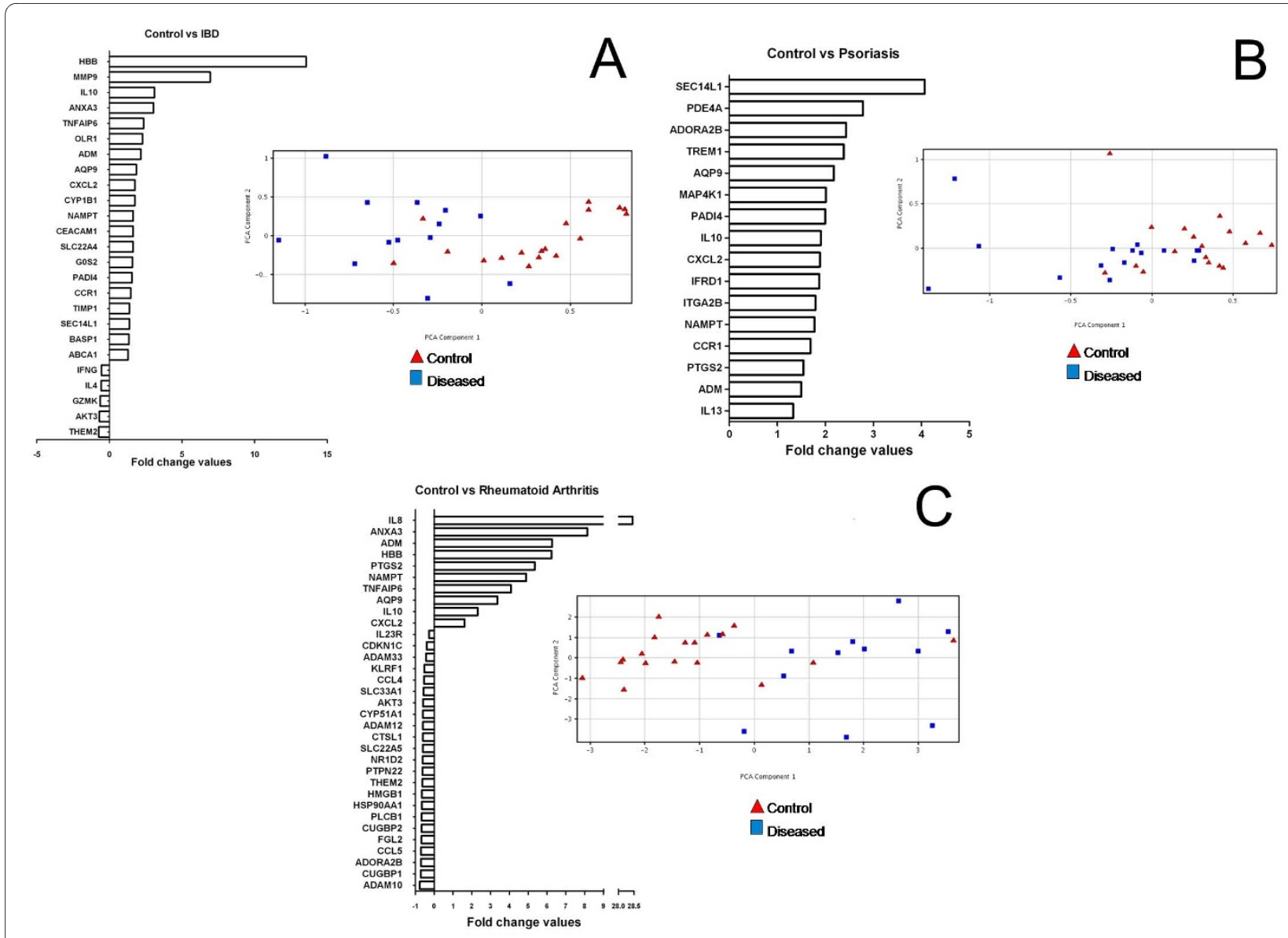

Figure 2 Fold changes of genes differentiating between diseased and control samples. Fold change values of genes, showing statistically significant (Mann-Whitney $U$ test) differential expression between diseased and control patients, were generated from RT-QPCR measurements and represent the difference of the means of the diseased and control groups. Principal component analysis was performed and separates the two groups of samples. $2 a$ represents the IBD, $2 \mathrm{~b}$ the psoriasis and $2 \mathrm{c}$ the RA gene panel.

Performing Gene Interaction Analysis resulted in the identification of a network that highlights the genes with the most interactions such as CCL5, IFNG, IL4, IL8, IL10, IL13, MMP9, PTGS2 and TIMP1 (Figure 3b) clearly showing that not only individual genes but entire networks are impacted and hence can be identified. Although there are genes that showed disease specific signatures, all of these genes showing significant differences between diseased and healthy samples form a network which might represent the common background of the pathogenesis of this type of inflammation.

As the next step in our analysis all the significantly changing genes were assigned to functional categories that were created based on EASE, the Expression Analysis Systematic Explorer that provides statistical methods for discovering biological themes within gene lists [17]. Genes were grouped into inflammatory response; cell growth and maintenance; proteolysis; metabolism and unclassified (including genes in unique categories or genes without categories) categories with 21,11, 7, 9 and 7 genes respectively. In order to illustrate the similarities in the pathogenetic background of these diseases, the functional categories, the number of genes and the disease gene panels are visualized. It shows that there are similar number of genes in the same functional categories in each of the diseases, though the number of unique genes (11 in IBD, 6 in psoriasis and 21 in RA) separating only a disease from the control group is also high (Figure $4)$.

\section{Diseases subtype stratification by differentially expressed genes}

We also sought to identify potential markers that differentiate between distinct subtypes or states of prognosis in each of the three diseases to stratify the disease based on gene expression patterns (Figure 5). Analyses of individual genes are provided in the supplement material Additional File 1: Figure S7-9. 

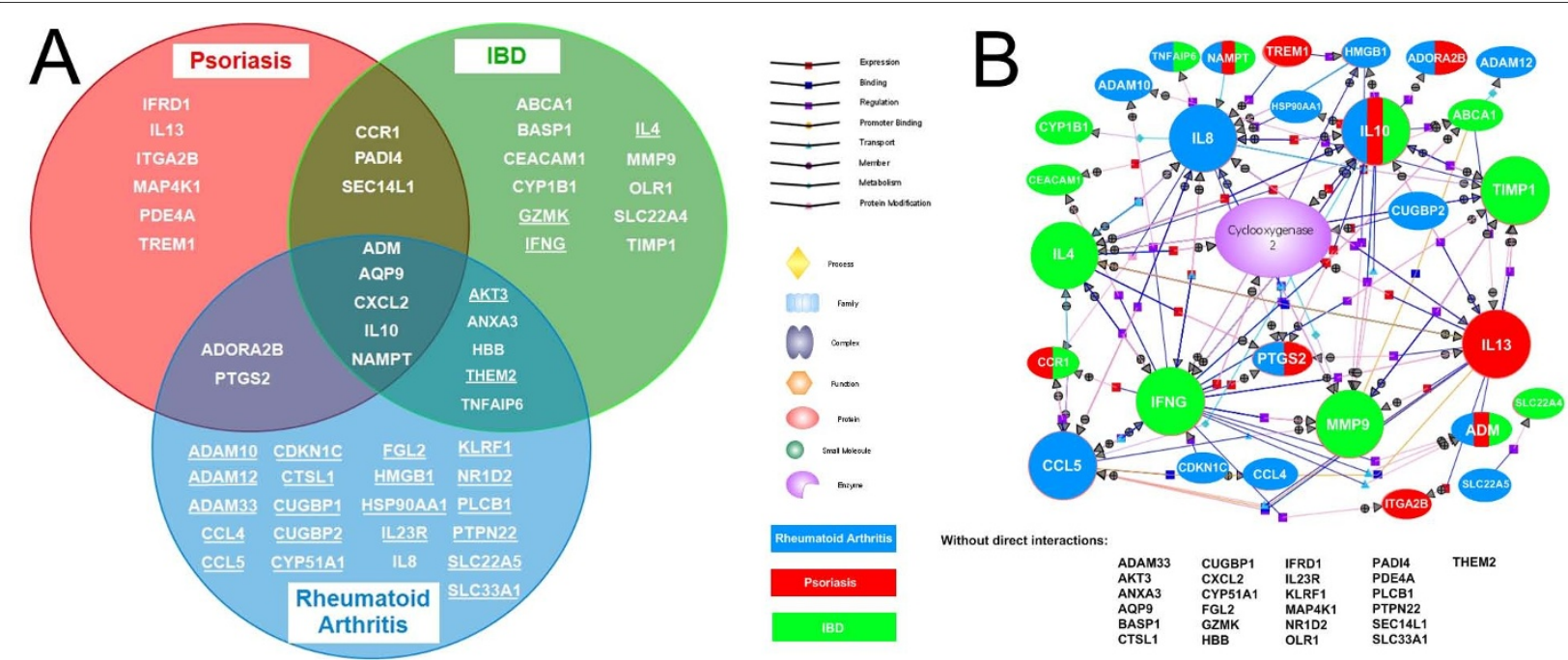

Figure 3 Genes separating chronic inflammatory diseases from controls. (a) Venn diagram shows all of those genes that show significant differences between each disease group and control samples. Each set contains genes that separate control samples only from the particular disease or diseases. Underlined genes were down-regulated compared to healthy controls. (b) Gene interaction analysis in GeneSpring GX in Direct Interactions mode highlights 28 genes that have direct interactions with each other while 25 genes have no direct interactions. Genes with the highest number of interactions are shown in extended size. Cyclooxygenase 2 enzyme is located in the middle of this pathway network. The genes that showed significant differences between one of the diseased groups and healthy controls have color codes in which blue represents RA, green represents IBD and red codes for psoriasis-related genes.

Regarding IBD, ADAM10, CUGBP1, CUGBP2, DUSP1, ETS2, FOS, IL6, IL18 and PGRMC1 show significantly higher expression levels in patients with CD compared to patients with UC (Figure 5a).

In psoriasis, CCL4, GZMK and KLRF1 show significantly higher, while ADM, ANXA3, IL4, MMP9 and OLR1 show significantly lower expression levels in patients with arthritis negative psoriasis compared to patients with symptoms of arthritis (Figure $5 \mathrm{~b}$ ).

HBB and PADI4 show lower; GZMK shows higher expression in RA patients with bone erosions confirmed with Magnetic Resonance Imaging compared to those without such symptoms (Figure 5c).

\section{Peripheral blood derived universal markers of chronic inflammation}

Importantly, we have identified five genes, ADM, AQP9, CXCL2, IL10 and NAMPT that show significant differences in expression levels between all the three conditions and control samples (Figure 6). These genes might be considered universal markers of chronic inflammation in PBMCs. Regarding pathway analysis that features the biological processes the genes are associated with; IL10 has the highest number of related biological processes (over 20) including lymphocyte, $\mathrm{T}$ and $\mathrm{B}$ cell proliferation, interleukin production and macrophage activation. $\mathrm{ADM}$ is related to 10 processes such as activation of MAPK-activity and interleukin-6 production; while AQP9, CXCL2 and NAMPT are only associated with a single process, urea transport, interleukin-18 production and neutrophil apoptosis, respectively (Figure 7).

We also determined the number of diseased patients with gene expression levels above the range of the mean + $\mathrm{SD}$ or $2 \mathrm{SD}$ of control samples. Additional File 1: Figure S6.

\section{Discussion}

It is of critical importance to identify new biomarkers and hints of mechanisms of disease states and disease progression in illnesses affecting large populations such as chronic inflammatory diseases in order to personalize treatment and accurately monitor disease progression. Methods based on genomic approaches and amongst them global gene expression profiles play an increasing role in such efforts.

The pathomechanism of these chronic inflammatory diseases has been examined at different levels including cell cultures, animal models and clinical samples. Importantly, as the molecular signature of disease across tissues is overall more prominent than the signature of tissue expression across diseases [18], there is good reason to believe one can detect disease-specific gene expression signatures in PBMC samples which are easy-to-access.

There have been studies analyzing the gene expression patterns of these diseases in PBMC samples which led to the identification of genes, which we also found to be significantly different between disease and control patients. Examples include PTGS2 in psoriasis [10]; PTPN22 in RA [19] and GZMK in IBD [12]. Other groups compared 

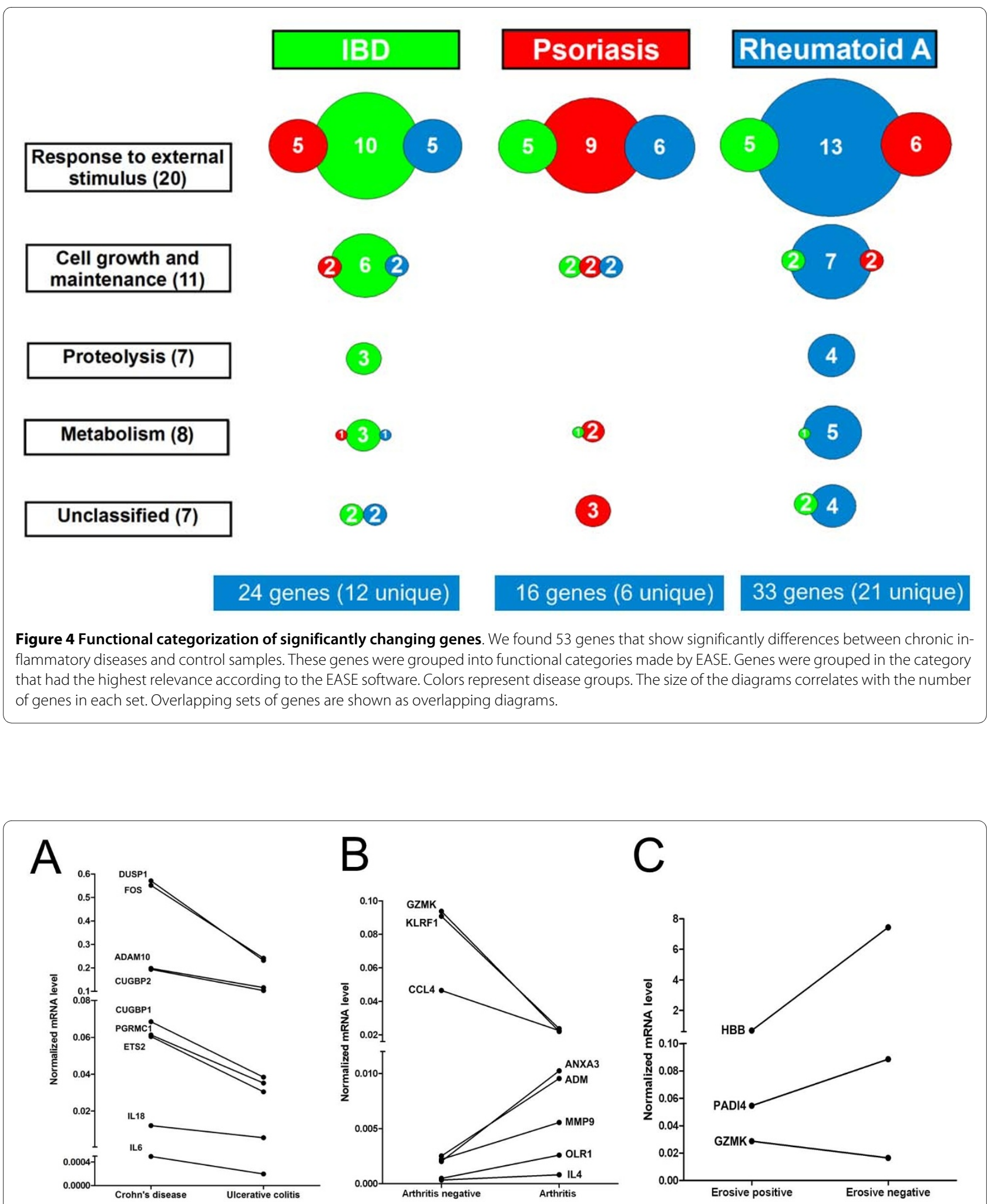

Figure 5 Correlation between gene expression levels and clinical parameters. Normalized mRNA levels of genes showing significant differences between patients with (a) Crohn's disease and Ulcerative colitis; (b) psoriasis patients with and without arthritis; (c) RA patients with and without MRconfirmed bone erosion were generated from RT-QPCR measurements and represent the means of the expression levels of the diseased and control groups. 


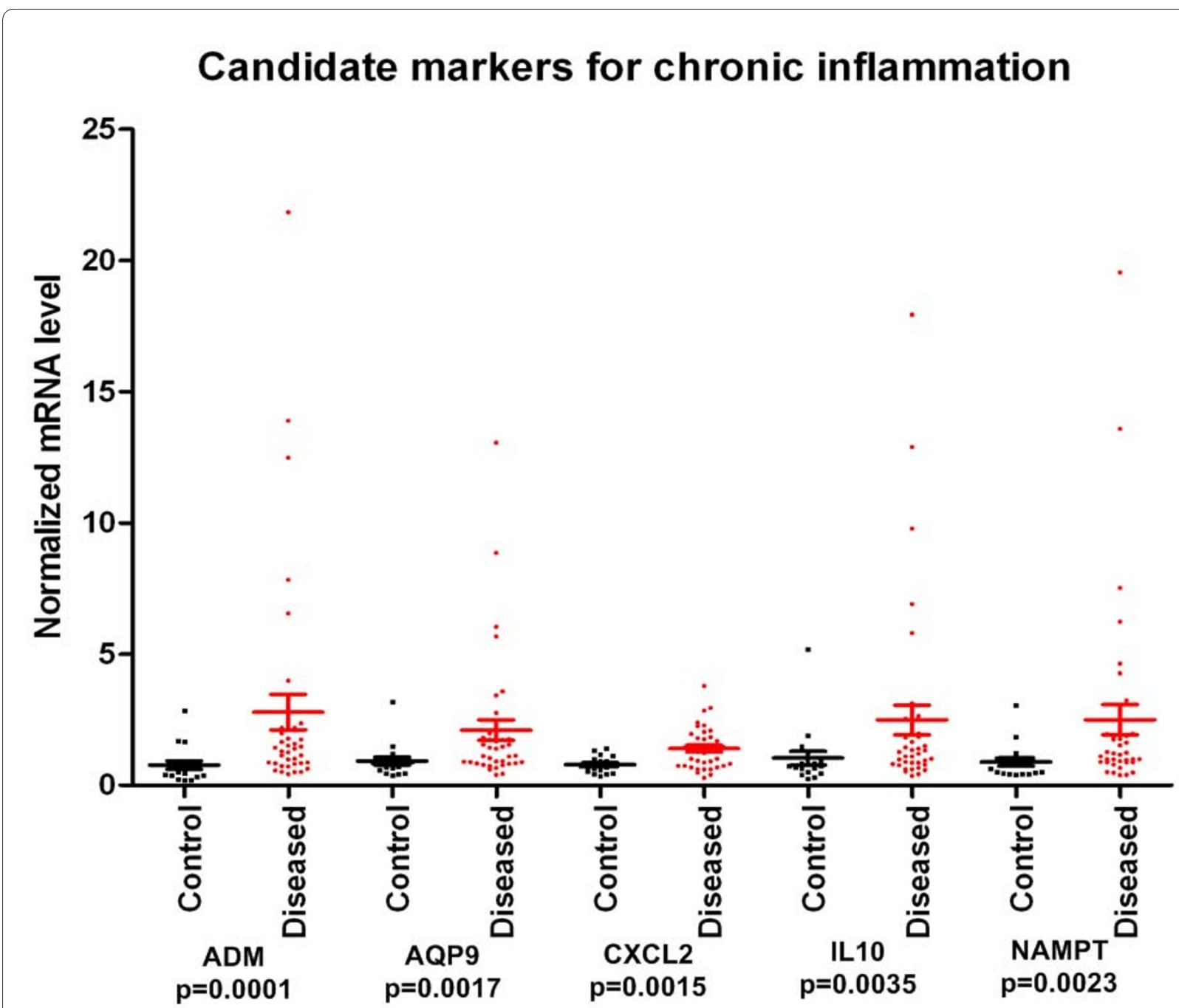

Figure 6 Peripheral blood derived universal markers of chronic inflammation. Normalized mRNA levels of genes (with SD) showing significant differences between all the samples of chronic inflammatory diseases and healthy controls were generated from RT-QPCR measurements.

different chronic disorders to each others such as juvenile arthritis and spondyloarthropathy [20] or psoriatic arthritis, RA and spondyloarthropathy [21], but such an extended number of genes have not yet been found in peripheral blood. Importantly the gene expression profiles of RA, IBD and psoriasis have not been compared previously. We tried to fill this gap with our study and identify markers of chronic inflammation that overlap among the 3 conditions and also ones that discriminate them from each other.

It should be noted though gene expression analyses of these diseases provide little insight into the pathomechanisms of the disease simply because it is impossible to sort out what is cause and what is consequence. However it can still provide hints or clues about the potential pathways affected. Therefore such datasets, although indirectly, can form the basis of more mechanistic studies.
This is why we have carried out and presented the gene interaction analyses of the genes identified and linked to the different diseases. Significantly the existence of overlapping gene sets suggests that there are common pathways impacted among the studied diseases. A common characteristic of all diseases is an altered inflammatory response.

In addition, genetic association studies have already identified SNPs linked to these diseases. A comparison of our dataset to these can provide further support to those findings [Table 1].

Several of the now identified differentially expressed genes have been linked to the studied diseases although either at a different level or different model. Regarding the IBD gene panel, OLR1/LOX1 [22] and CCR1 [23] both have immune response function and are expressed in human intestinal cells. The homophilic ligation of 


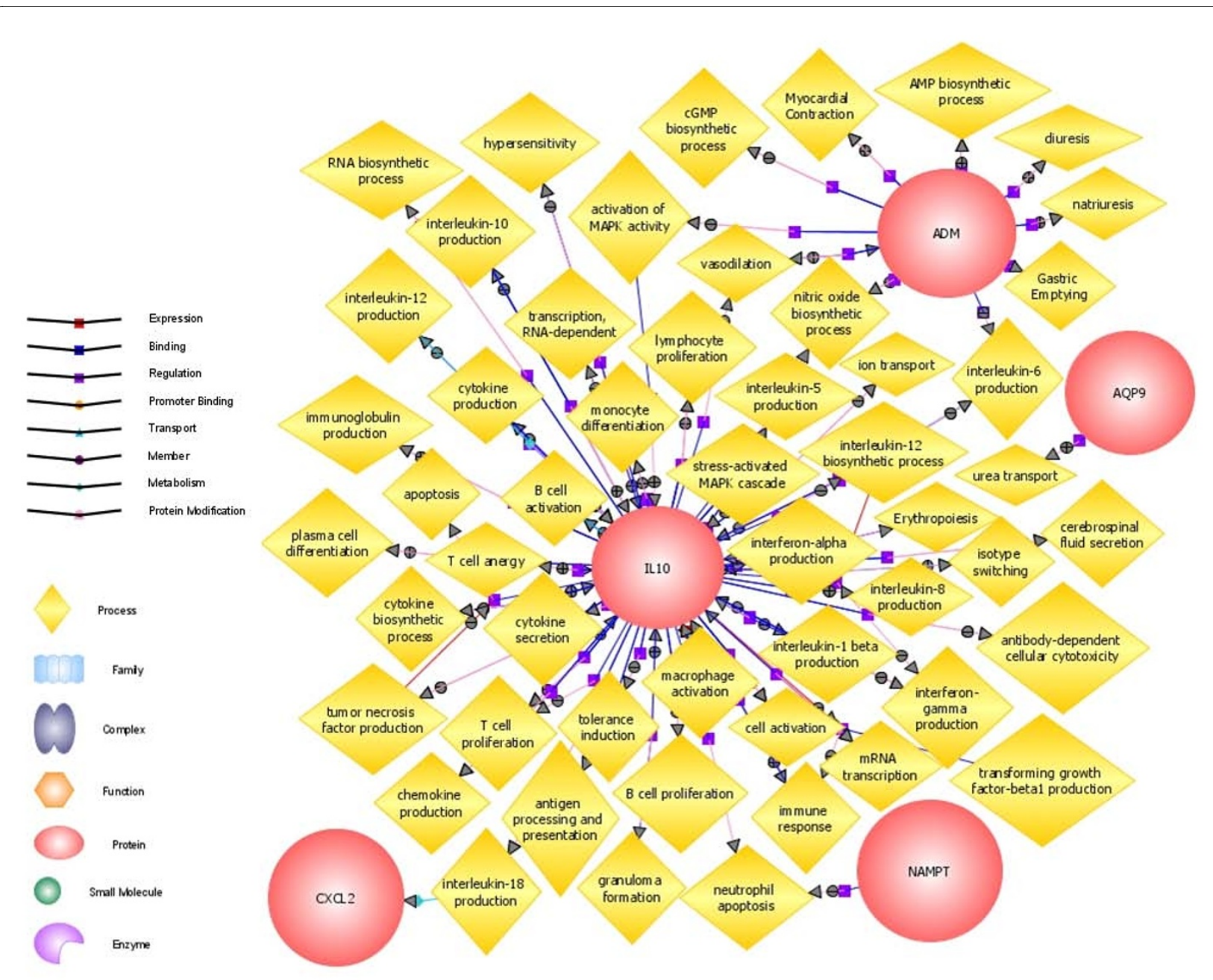

Figure 7 Pathway analysis of universal markers of chronic inflammation. Pathway analysis was carried out with GeneSpring GX Biological Processes mode that revealed all the biological processes the genes are related to. Circles represent the 5 genes; deltoids represent biological processes.

CEACAM1, which is a cell-surface molecule, on T cells leads to the inhibition of a range of $\mathrm{T}$-cell functions therefore it might be a new potential therapeutic target in the treatment of IBD [24]. MMP9, a gelatinase, that showed a 7 -fold up-regulation in diseased samples in our study is an important mediator of tissue injury in colitis and is upregulated in sputum samples of CD patients [25]. Garg et al. suggested that developing strategies to block MMP-9 activity in the gut might be of benefit to IBD [26]. TIMP1 is a natural inhibitor of matrix metalloproteinases (MMPs) and might be considered a potential drug target. The DNA methylation of IFNG that regulates immune response was analyzed within the mucosal compartment in both normal and IBD populations [27]. PTGS2/COX2 is related to IBD expression level in colon biopsy [28]. ABCA1, BASP1, GZMK, HBB, SEC14L1, THEM2 and TNFAIP6 have never been associated with IBD; therefore further analyses should be carried out.
In the psoriasis panel, CCR1 that plays a role in inflammatory response is associated with psoriasis via the analysis of skin lesion samples [29]. Koczan et al. identified pairs of genes such as PTGS2/COX2 and NR1D2 which allowed an accuracy of disease stage prediction of $86 \%$, based on gene expression patterns [10]. ADORA2B, IFRD1, ITGA2B, MAP4K1, PADI4, PDE4A, SEC14L1 and TREM1 have no documented association with psoriasis yet. Therefore these are candidates for further SNP/ genetic association or mechanistic studies.

Regarding the significantly changing genes in RA samples, IL8 is related to RA based on expression [30]. Van Der Voort et al. demonstrated that the expression of ADAM10 is strongly enhanced in RA synovia [31]. CCL4 was expressed in PBMC [30] and CCL5 showed high serum levels [32]. Kehlen et al. found up-regulation of TNFAIP6 in fibroblast-like synoviocytes of patients with RA [33]. HMGB1 [34] and PLCB1 [35] only showed dif- 
ference in protein level in RA patients. PTGS2/COX2 mRNA levels in PBMC samples from RA patients were within the normal range or below normal [36]. ADAM12, ADAM33, ADORA2B, AKT3, ANXA3, CDKN1C, CTSL1, CUGBP1, CUGBP2, CYP51A1, FGL2, HBB, HSP90AA1, KLRF1, NR1D2, SLC33A1 and THEM2 have no evidence of association with RA and should further be studied.

Gene expression profiling may allow early diagnosis, aid in identifying prognosis or subtypes. We found genes that differentiate between CD and UC in IBD of which some have already been associated with the disease but at a different level or in a different tissue. Alexander et al. reported that the expression level of FOS was two- to threefold higher in involved than in uninvolved areas of the colons of two UC patients [37]. van der Pouw Kraan at el. found that ETS2 may be an important transcription factor driving inflammation in acute as well as chronic inflammatory diseases such as IBD [38]. As oppose to UC, IL-18 may serve as indicators of acute phase reactivity in CD, according to Haas et al. [39], that correlates with our findings. Mitsuyama et al. suggested that IL-6 trans-signaling may play role in the development of IBD and imply the possibility of a selective therapeutic strategy to target this signaling [40].

A gene panel containing 8 genes shows significant differences between patients with and without arthritis in psoriasis. Hitchon et al. described that MMPs, especially MMP2 and MMP9, have been implicated in several features of inflammatory arthritis including angiogenesis and bone erosions [41]. Partsch et al. measured the protein level of IL4 in synovial fluid of patients with psoriatic arthritis (PsA) [42].

Another gene set including HBB, GZMK and PADI4 separates different states of prognosis in RA regarding MR-confirmed bone erosion. In RA, PADI4 is a target of autoantibodies; and its increased expression in synovia of RA patients [43], also its relation to the disease at SNP level have already been confirmed [44]. Osteoclasts are shown to be mainly involved in the bone-destruction of RA which may indicate further studies regarding the role of PADI4 in bone erosion.

We consider a key aspect of our work is the identification of five genes including ADM, AQP9, CXCL2, IL10 and NAMPT that differentiate between samples from patients with chronic inflammation and healthy controls. ADM that plays role in response to wounding is found to be distributed on the surface of the human colonic mucosa [45] and the plasma level of ADM in RA patients was significantly higher compared to healthy controls in synovial tissue [46]. CXCL2 that has function in immune response is over-expressed in osteoarthritis fibroblasts rather than rheumatoid fibroblasts [47], up-regulated in psoriatic epidermis [48], and also in epithelial tissue [49].
IL10 is expressed in PBMCs of psoriasis [50], RA [51] and IBD patients [52]. The expression of NAMPT which is a pre-B cell-enhancing factor is increased in colonic biopsy specimens of IBD patients compared to healthy controls [53], it is also up-regulated in plasma and synovial fluid of RA patients [54]; and in PBMC of psoriasis in the diseased stage [10]. AQP9 that has an activity in immune response has never been associated with any of these medical conditions.

These genes might serve as universal markers of chronic inflammation. As expected, IL10 seems to be the key gene in this network regarding biological processes (Figure $3 \mathrm{~b}$ ). As the number of biological processes related to ADM is relatively high and it has no direct interactions with the genes we examined, there might be other potential markers that play role in the pathomechanism of the conditions we analyzed that also have similar pathways or targets as ADM. Chronic inflammatory and autoimmune diseases share a number of phenotypic and genetic characteristics suggesting common etiological pathways or pathomechanisms. Becker et al. used meta-analyses of whole-genome scans and found non-random clustering of disease susceptibility loci for autoimmune diseases [55]. Our results may suggest that all of the chronic inflammatory conditions we analyzed share similar pathogenetic background as reflected by peripheral gene expression.

Personalized medicine is becoming an integral part of healthcare and the key challenge is to establish a strategic focus on biomarker-based clinical tests. Non-invasive or minimally invasive diagnostic tests present less danger to a patient than invasive tests such as biopsies; and can help clinicians by providing them with valuable information in decision-making. A biomarker assay based on the gene panels described above might save time by reducing a list of preliminary disease impressions to a definitive diagnosis. The evaluation of these results and the identification of genes with altered expression or marker genes could also be potential targets for novel and more effective therapies and may lead to important insights into the pathogenesis of chronic inflammatory diseases. It might also have benefits to look at the identified gene panels in terms of SNPs in order to identify potential genetic changes associated with these medical conditions. Such novel and known biomarkers or a panel of such markers can play a major role in the development of personalized medicine.

\section{Conclusion}

Our results suggest that IBD, psoriasis and rheumatoid arthritis have common pathogenetic background and gene expression profiling from peripheral blood might reveal novel targets and pathways affected by these diseases. 


\section{Additional material}

Additional file 1 List of genes, gene interaction analyses and detailed gene expression data. Figure S1) List of genes that were measured on the TaqMan Low Density Arrays. • Figure S2) Inclusion and exclusion criteria of diseased patients and healthy controls. Figure S3) Gene interaction analysis ("Cell mode" in Genespring GX) of the genes differentiating between IBD and controls. • Figure S4) Gene interaction analysis ("Cell mode" in GeneSpring GX) of the genes differentiating between psoriasis and controls. • Figure S5) Gene interaction analysis ("Cell mode" in GeneSpring GX) of the genes differentiating between RA and controls. • Figure S6) Number of diseased patients with gene expression levels outside the range of the mean \pm SD or 2 SD in control sample. Based on Figure 6 . Figure S7) Stratifying IBD: The results of the RT-QPCR measurements for each gene that showed significant differences between Crohn's disease vs Ulcerative colitis patients. Figure S8) Stratifying Psoriasis: The results of the RTQPCR measurements for each gene that showed significant differences between arthritis negative vs positive forms. - Figure 59) Stratifying RA: The results of the RT-QPCR measurements for each gene that showed significant differences between patients with MRI confirmed bone erosion vs patients without bone erosion.

\section{Abbreviations}

ABCA1: ATP-binding cassette, sub-family A, member 1; ADAM10: ADAM metallopeptidase domain 10; ADAM12: ADAM metallopeptidase domain 12; ADAM33: ADAM metallopeptidase domain 33; ADM: Adrenomedullin ADORA2B: adenosine A2b receptor; AKT3: v-akt murine thymoma viral oncogene homolog 3; ANXA3: annexin A3; AQP9: Aquaporin 9; BASP1: brain abundant, membrane attached signal protein 1; CCL4: chemokine (C-C motif) ligand 4; CCL5: chemokine (C-C motif) ligand 5; CCR1: chemokine (C-C motif) receptor 1; CDKN1C: cyclin-dependent kinase inhibitor 1C; CEACAM1: carcinoembryonic antigen-related cell adhesion molecule 1; CTSL1: cathepsin L1; CUGBP1: CUG triplet repeat, RNA binding protein 1; CUGBP2: CUG triplet repeat, RNA binding protein 2; CXCL2: chemokine (C-X-C motif) ligand 2; CYP1B1: cytochrome P450, family 1, subfamily B, polypeptide 1; CYP51A1: cytochrome P450, family 51, subfamily A, polypeptide 1; FGL2: fibrinogen-like 2; GK: glycerol kinase; GZMK: granzyme K; HBB: hemoglobin, beta; HSP90AA1: heat shock protein $90 \mathrm{kDa}$ alpha (cytosolic), class A member 1; IBD: Inflammatory bowel disease; IFNG: interferon gamma; IFRD1: interferon-related developmental regulator 1; IL10: Interleukin 10; IL13: Interleukin 13; IL23R: Interleukin 23 receptor; IL4: Interleukin 4; IL8: Interleukin 8; ITGA2B: integrin, alpha 2b; KLRF1: killer cell lectin-like receptor subfamily $F$, member 1 ; MAP4K1: mitogen-activated protein kinase 1; MMP9: matrix metallopeptidase 9; NAMPT: nicotinamide phosphoribosyltransferase; NR1D2: nuclear receptor subfamily 1, group D, member 2; OLR1: oxidized low density lipoprotein receptor 1; PADI4: peptidyl arginine deiminase, type IV; PBMC: Peripheral Blood Mononuclear Cells; PDE4A: CAMP-specific phosphodiesterase 4D; PLCB1: phospholipase C, beta 1 (phosphoinositide-specific); PTGS2: prostaglandin-endoperoxide synthase 2 (prostaglandin G/H synthase and cyclooxygenase); PTPN22: protein tyrosine phosphatase, non-receptor type 22 (lymphoid); QPCR: Quantitative polymerase chain reaction; RA: Rheumatoid arthritis; SEC14L 1: SEC14-like 1 (S. cerevisiae); SLC22A4: solute carrier family 22 member 4; SLC22A5: solute carrier family 22 member 5; SLC33A1: solute carrier family 33 member 1; THEM2: acylCOA thioesterase 13; TIMP1: TIMP metallopeptidase inhibitor 1; TLDA: Taqman Low Density Array; TNFAIP6: tumor necrosis factor, alpha-induced protein 6; TREM1: triggering receptor expressed on myeloid cells 1

\section{Competing interests}

The authors declare that they have no competing interests.

\section{Authors' contributions}

B.M designed and carried out experiments, analyzed data and wrote the paper. S.P. designed and carried out experiments and analyzed the data, A.S., Z.S., K.P. and M.P. determined patient inclusion and exclusion criteria, carried out patient recruitment and collection. L.N. directed research, designed experiments, analyzed the data and wrote the paper.

\section{Acknowledgements}

The authors are indebted to Ms. Zsuzsa Bodnar for clinical coordination. They also thank Dr Arpad Lanyi and the members of the Nagy laboratory for their comments. The authors would like to express their thanks for the expert technical assistance of Ms. Ibolya Furtos.

L.N is an International Scholar of HHMI and holds a Wellcome Trust Senior Research Fellowship in Biomedical Sciences, this work was also supported by grants from the National Office for Research and Technology (NKFP 1/007/01, NKFP 1A/008/04), from the National Research Fund (NI 67877) and a grant from the Hungarian Ministry of Health (ETT 294-07) to L.N.

\section{Author Details}

'Department of Biochemistry and Molecular Biology, University of Debrecen, Debrecen, Hungary, ${ }^{2}$ Apoptosis and Genomics Research Group of the Hungarian Academy of Sciences, Research Center for Molecular Medicine, Medical and Health Science Center, University of Debrecen, Debrecen, Hungary, ${ }^{3}$ Department of Dermatology, Medical and Health Science Center, University of Debrecen, Debrecen, Hungary, ${ }^{4}$ Clinical Genomics Center, Medical and Health Science Center, University of Debrecen, Debrecen, Hungary, ${ }^{5}$ Department of Rheumatology, Institute of Medicine, University of Debrecen, Medical and Health Science Center, Debrecen, Hungary and ${ }^{6} 2$ nd Department of Medicine, University of Debrecen, Medical and Health Science Center, Debrecen, Hungary

Received: 24 November 2009 Accepted: 5 May 2010 Published: 5 May 2010

\section{References}

1. Silman AJ, Pearson JE: Epidemiology and genetics of rheumatoid arthritis. Arthritis Res 2002, 4(Suppl 3):S265-272.

2. Langley RG, Krueger GG, Griffiths CE: Psoriasis: epidemiology, clinical features, and quality of life. Ann Rheum Dis 2005, 64(Suppl 2):ii18-23. discussion ii24-15

3. Loftus EV Jr: Clinical epidemiology of inflammatory bowel disease: Incidence, prevalence, and environmental influences. Gastroenterology 2004, 126(6):1504-1517.

4. Wu F, Dassopoulos T, Cope L, Maitra A, Brant SR, Harris ML, Bayless TM, Parmigiani G, Chakravarti S: Genome-wide gene expression differences in Crohn's disease and ulcerative colitis from endoscopic pinch biopsies: insights into distinctive pathogenesis. Inflamm Bowel Dis 2007, 13(7):807-821.

5. Nomura I, Gao B, Boguniewicz M, Darst MA, Travers JB, Leung DY: Distinct patterns of gene expression in the skin lesions of atopic dermatitis and psoriasis: a gene microarray analysis. J Allergy Clin Immunol 2003, 112(6):1195-1202.

6. van Baarsen LG, Wijbrandts CA, Timmer TC, Pouw Kraan TC van der, Tak PP, Verweij CL: Synovial tissue heterogeneity in rheumatoid arthritis in relationship to disease activity and biomarkers in peripheral blood. Arthritis Rheum 2010 in press.

7. Aziz H, Zaas A, Ginsburg GS: Peripheral blood gene expression profiling for cardiovascular disease assessment. Genomic Med 2007, 1(34):105-112.

8. Ma XJ, Salunga R, Tuggle JT, Gaudet J, Enright E, McQuary P, Payette T, Pistone M, Stecker K, Zhang BM, et al:: Gene expression profiles of human breast cancer progression. Proc Natl Acad Sci USA 2003, 100(10):5974-5979.

9. Maas K, Chan S, Parker J, Slater A, Moore J, Olsen N, Aune TM: Cutting edge: molecular portrait of human autoimmune disease. $\mathrm{J}$ Immunol 2002, 169(1):5-9.

10. Koczan D, Guthke R, Thiesen HJ, Ibrahim SM, Kundt G, Krentz H, Gross G, Kunz M: Gene expression profiling of peripheral blood mononuclear leukocytes from psoriasis patients identifies new immune regulatory molecules. Eur J Dermatol 2005, 15(4):251-257.

11. Junta CM, Sandrin-Garcia P, Fachin-Saltoratto AL, Mello SS, Oliveira RD, Rassi DM, Giuliatti S, Sakamoto-Hojo ET, Louzada-Junior P, Donadi EA, et al:: Differential gene expression of peripheral blood mononuclear cells from rheumatoid arthritis patients may discriminate immunogenetic, pathogenic and treatment features. Immunology 2009, 127(3):365-372.

12. Burczynski ME, Peterson RL, Twine NC, Zuberek KA, Brodeur BJ, Casciotti L, Maganti V, Reddy PS, Strahs A, Immermann F, et al:: Molecular classification of Crohn's disease and ulcerative colitis patients using transcriptional profiles in peripheral blood mononuclear cells. J Mol Diagn 2006, 8(1):51-61. 
13. Steinbach D, Schramm A, Eggert A, Onda M, Dawczynski K, Rump A, Pastan I, Wittig S, Pfaffendorf N, Voigt A, et al: Identification of a set of seven genes for the monitoring of minimal residual disease in pediatric acute myeloid leukemia. Clin Cancer Res 2006, 12(8):2434-2441.

14. Schobesberger M, Baltzer A, Oberli A, Kappeler A, Gugger M, Burger H Jaggi R: Gene expression variation between distinct areas of breast cancer measured from paraffin-embedded tissue cores. BMC Cancer 2008, 8:343.

15. Swingler TE, Waters JG, Davidson RK, Pennington CJ, Puente XS, Darrah C, Cooper A, Donell ST, Guile GR, Wang W, et al:: Degradome expression profiling in human articular cartilage. Arthritis Res Ther 2009, 11(3):R96.

16. Kosa JP, Balla B, Kiss J, Podani J, Takacs I, Lazary A, Nagy Z, Bacsi K, Karsai A, Speer $G$, et al:: Postmenopausal expression changes of immune systemrelated genes in human bone tissue. J Clin Immunol 2009, 29(6):761-768.

17. Hosack DA, Dennis G Jr, Sherman BT, Lane HC, Lempicki RA: Identifying biological themes within lists of genes with EASE. Genome Biol 2003, 4(10):R70.

18. Dudley JT, Tibshirani R, Deshpande T, Butte AJ: Disease signatures are robust across tissues and experiments. Mol Syst Biol 2009, 5:307.

19. Batliwalla FM, Baechler EC, Xiao X, Li W, Balasubramanian S, Khalili H, Damle A, Ortmann WA, Perrone A, Kantor AB, et al:: Peripheral blood gene expression profiling in rheumatoid arthritis. Genes Immun 2005 6(5):388-397.

20. Barnes MG, Aronow BJ, Luyrink LK, Moroldo MB, Pavlidis P, Passo MH, Grom AA, Hirsch R, Giannini EH, Colbert RA, et al:: Gene expression in juvenile arthritis and spondyloarthropathy: pro-angiogenic ELR+ chemokine genes relate to course of arthritis. Rheumatology (Oxford) 2004, 43(8):973-979.

21. Gu J, Marker-Hermann E, Baeten D, Tsai WC, Gladman D, Xiong M, Deister $H$, Kuipers JG, Huang F, Song YW, et al:: A 588-gene microarray analysis of the peripheral blood mononuclear cells of spondyloarthropathy patients. Rheumatology (Oxford) 2002, 41(7):759-766.

22. Bruneau N, Richard S, Silvy F, Verine A, Lombardo D: Lectin-like Ox-LDL receptor is expressed in human INT-407 intestinal cells: involvement in the transcytosis of pancreatic bile salt-dependent lipase. Mol Biol Cell 2003, 14(7):2861-2875

23. Dwinell MB, Eckmann L, Leopard JD, Varki NM, Kagnoff MF: Chemokine receptor expression by human intestinal epithelial cells. Gastroenterology 1999, 117(2):359-367.

24. Nagaishi T, Chen Z, Chen L, lijima H, Nakajima A, Blumberg RS: CEACAM and the regulation of mucosal inflammation. Mucosal Immunol 2008, 1(Suppl 1):S39-42

25. Fireman E, Kraiem Z, Sade O, Greif J, Fireman Z: Induced sputumretrieved matrix metalloproteinase 9 and tissue metalloproteinase inhibitor 1 in granulomatous diseases. Clin Exp Immunol 2002, 130(2):331-337.

26. Garg P, Vijay-Kumar M, Wang L, Gewirtz AT, Merlin D, Sitaraman SV: Matrix metalloproteinase-9-mediated tissue injury overrides the protective effect of matrix metalloproteinase-2 during colitis. Am J Physiol Gastrointest Liver Physiol 2009, 296(2):G175-184.

27. Gonsky R, Deem RL, Targan SR: Distinct Methylation of IFNG in the Gut. $J$ Interferon Cytokine Res 2009, 29(7):407-414.

28. Hendel J, Nielsen OH: Expression of cyclooxygenase-2 mRNA in active inflammatory bowel disease. Am J Gastroenterol 1997, 92(7):1170-1173.

29. Rottman JB, Smith TL, Ganley KG, Kikuchi T, Krueger JG: Potential role of the chemokine receptors CXCR3, CCR4, and the integrin alphaEbeta7 in the pathogenesis of psoriasis vulgaris. Lab Invest 2001, 81(3):335-347.

30. Koczan D, Drynda S, Hecker M, Drynda A, Guthke R, Kekow J, Thiesen HJ: Molecular discrimination of responders and nonresponders to antiTNF alpha therapy in rheumatoid arthritis by etanercept. Arthritis Res Ther 2008, 10(3):R50.

31. Voort R van der, van Lieshout AW, Toonen LW, Sloetjes AW, Berg WB van den, Figdor CG, Radstake TR, Adema GJ: Elevated CXCL16 expression by synovial macrophages recruits memory $\mathrm{T}$ cells into rheumatoid joints. Arthritis Rheum 2005, 52(5):1381-1391.

32. Yang MH, Wu FX, Xie CM, Qing YF, Wang GR, Guo XL, Tang Z, Zhou JG, Yuan GH: Expression of CC chemokine ligand 5 in patients with rheumatoid arthritis and its correlation with disease activity and medication. Chin Med SciJ 2009, 24(1):50-54.

33. Kehlen A, Pachnio A, Thiele K, Langner J: Gene expression induced by interleukin-17 in fibroblast-like synoviocytes of patients with rheumatoid arthritis: upregulation of hyaluronan-binding protein TSG6. Arthritis Res Ther 2003, 5(4):R186-192.

34. Zuo XX, Zhou YO, Gong YH, Wang YP, Tang DL, Xiao XZ: [Expression of high mobility group box chromosomal protein 1 in peripheral blood of patients with rheumatoid arthritis]. Zhonghua Nei Ke Za Zhi 2007, 46(7):547-550

35. Zini N, Lisignoli G, Solimando L, Bavelloni A, Grassi F, Guidotti L, Trimarchi C, Facchini A, Maraldi NM: IL1-beta and TNF-alpha induce changes in the nuclear polyphosphoinositide signalling system in osteoblasts similar to that occurring in patients with rheumatoid arthritis: an immunochemical and immunocytochemical study. Histochem Cell Biol 2003, 120(3):243-250.

36. Schulze-Koops H, Davis LS, Kavanaugh AF, Lipsky PE: Elevated cytokine messenger RNA levels in the peripheral blood of patients with rheumatoid arthritis suggest different degrees of myeloid cell activation. Arthritis Rheum 1997, 40(4):639-647.

37. Alexander RJ, Panja A, Kaplan-Liss E, Mayer L, Raicht RF: Expression of protooncogene-encoded mRNA by colonic epithelial cells in inflammatory bowel disease. Dig Dis Sci 1996, 41 (4):660-669.

38. Pouw Kraan TC van der, Zwiers A, Mulder CJ, Kraal G, Bouma G: Acute experimental colitis and human chronic inflammatory diseases share expression of inflammation-related genes with conserved Ets 2 binding sites. Inflamm Bowel Dis 2009, 15(2):224-235.

39. Haas SL, Abbatista M, Brade J, Singer MV, Bocker U: Interleukin-18 serum levels in inflammatory bowel diseases: correlation with disease activity and inflammatory markers. Swiss Med Wkly 2009, 139(9-10):140-145.

40. Mitsuyama K, Sata M, Rose-John S: Interleukin-6 trans-signaling in inflammatory bowel disease. Cytokine Growth Factor Rev 2006, 17(6):451-461

41. Hitchon CA, Danning CL, Illei GG, El-Gabalawy HS, Boumpas DT: Gelatinase expression and activity in the synovium and skin of patients with erosive psoriatic arthritis. J Rheumatol 2002, 29(1):107-117.

42. Partsch G, Wagner E, Leeb BF, Broll H, Dunky A, Smolen JS: T cell derived cytokines in psoriatic arthritis synovial fluids. Ann Rheum Dis 1998, 57(11):691-693

43. Chang $X$, Zhao Y, Sun S, Zhang Y, Zhu Y: The expression of PADI4 in synovium of rheumatoid arthritis. Rheumatol Int 2009, 29(12):1411-6.

44. Li Y, Begovich AB: Unraveling the genetics of complex diseases: Susceptibility genes for rheumatoid arthritis and psoriasis. Semin Immunol 2009, 21(6):318-27.

45. Marutsuka K, Nawa Y, Asada Y, Hara S, Kitamura K, Eto T, Sumiyoshi A: Adrenomedullin and proadrenomudullin $\mathrm{N}$-terminal 20 peptide (PAMP) are present in human colonic epithelia and exert an antimicrobial effect. Exp Physio/ 2001, 86(5):543-545.

46. Chosa E, Hamada H, Kitamura K, Eto T, Tajima N: Increased plasma and joint tissue adrenomedullin concentrations in patients with rheumatoid arthritis compared to those with osteoarthritis. Rheumatol 2003, 30(12):2553-2556.

47. Scaife S, Brown R, Kellie S, Filer A, Martin S, Thomas AM, Bradfield PF, Amft $\mathrm{N}$, Salmon M, Buckley CD: Detection of differentially expressed genes in synovial fibroblasts by restriction fragment differential display. Rheumatology(Oxford) 2004, 43(11):1346-1352.

48. Nukui T, Ehama R, Sakaguchi M, Sonegawa H, Katagiri C, Hibino T, Huh NH: S100A8/A9, a key mediator for positive feedback growth stimulation of normal human keratinocytes. J Cell Biochem 2008, 104(2):453-464.

49. Wu F, Zikusoka M, Trindade A, Dassopoulos T, Harris ML, Bayless TM, Brant SR, Chakravarti S, Kwon JH: MicroRNAs are differentially expressed in ulcerative colitis and alter expression of macrophage inflammatory peptide-2 alpha. Gastroenterology 2008, 135(5):1624-1635. e1624

50. Fits L Van Der, Kant M, Wel LI Van Der, Prens EP: Polymorphisms in the interferon regulatory factor-1 promoter are not associated with psoriasis and do not influence IFN-alpha-induced Th1 polarization. $J$ Interferon Cytokine Res 2007, 27(10):841-846.

51. Bucht A, Larsson P, Weisbrot L, Thorne C, Pisa P, Smedegard G, Keystone EC, Gronberg A: Expression of interferon-gamma (IFN-gamma), IL-10, IL12 and transforming growth factor-beta (TGF-beta) mRNA in synovial fluid cells from patients in the early and late phases of rheumatoid arthritis (RA). Clin Exp Immunol 1996, 103(3):357-367.

52. Gasche C, Bakos S, Dejaco C, Tillinger W, Zakeri S, Reinisch W: IL-10 secretion and sensitivity in normal human intestine and inflammatory bowel disease. J Clin Immunol 2000, 20(5):362-370. 
53. Moschen AR, Kaser A, Enrich B, Mosheimer B, Theurl M, Niederegger $H$, Tilg H: Visfatin, an adipocytokine with proinflammatory and immunomodulating properties. J Immunol 2007, 178(3):1748-1758.

54. Nowell MA, Richards PJ, Fielding CA, Ognjanovic S, Topley N, Williams AS, Bryant-Greenwood G, Jones SA: Regulation of pre-B cell colonyenhancing factor by STAT-3-dependent interleukin- 6 trans-signaling: implications in the pathogenesis of rheumatoid arthritis. Arthritis Rheum 2006, 54(7):2084-2095.

55. Becker KG: Comparative genetics of type 1 diabetes and autoimmune disease: common loci, common pathways? Diabetes 1999, 48(7):1353-1358.

\section{Pre-publication history}

The pre-publication history for this paper can be accessed here: http://www.biomedcentral.com/1755-8794/3/15/prepub

doi: $10.1186 / 1755-8794-3-15$

Cite this article as: Mesko et al., Peripheral blood gene expression patterns discriminate among chronic inflammatory diseases and healthy controls and identify novel targets BMC Medical Genomics 2010, 3:15

Submit your next manuscript to BioMed Central and take full advantage of:

- Convenient online submission

- Thorough peer review

- No space constraints or color figure charges

- Immediate publication on acceptance

- Inclusion in PubMed, CAS, Scopus and Google Scholar

- Research which is freely available for redistribution

Submit your manuscript at www.biomedcentral.com/submit
C) Biomed Central 